\title{
Nambu-Goldstone boson hypothesis for squarks and sleptons in pure gravity mediation
}

\author{
Tsutomu T. Yanagida, ${ }^{a}$ Wen Yin $^{b}$ and Norimi Yokozaki ${ }^{b}$ \\ ${ }^{a}$ Kavli Institute for the Physics and Mathematics of the Universe (WPI), \\ University of Tokyo, \\ Kashiwa 277-8583, Japan \\ ${ }^{b}$ Department of Physics, Tohoku University, \\ Sendai, Miyagi 980-8578, Japan \\ E-mail: tsutomu.tyanagida@ipmu.jp, yinwen@tuhep.phys.tohoku.ac.jp, \\ yokozaki@truth.phys.tohoku.ac.jp
}

ABSTRACT: We point out that a hypothesis of squarks and sleptons being NambuGoldstone (NG) bosons is consistent with pure gravity mediation or minimal split supersymmetry (SUSY). As a concrete example, we consider a SUSY $E_{7} / \mathrm{SU}(5) \times \mathrm{U}(1)^{3}$ non-linear sigma model in the framework of pure gravity mediation. The model accommodates three families of the quark and lepton chiral multiplets as (pseudo) NG multiplets of the Kähler manifold, which may enable us to understand the origin and number of the families. We point out that squarks in the first and second generations are likely to be as light as a few $\mathrm{TeV}$ if the observed baryon asymmetry is explained by the thermal leptogenesis; therefore, these colored particles can be discovered at the LHC Run-2 or at the high luminosity LHC.

KEYWORDS: Supersymmetry Phenomenology

ARXIV EPRINT: 1608.06618 


\section{Contents}

1 Introduction 1

$2 \quad E_{7} / \mathrm{SU}(5) \times \mathrm{U}(1)^{3}$ model in pure gravity mediation 2

3 Phenomenological implications $\quad 4$

3.1 Case of vanishing squark and slepton masses 4

3.2 Case of non-vanishing squark and slepton masses 11

$\begin{array}{lll}3.3 \text { Mass spectra } & 12\end{array}$

4 Conclusion $\quad 13$

A Vanishing soft mass for NG multiplet with direct coupling to the SUSY $\begin{array}{ll}\text { breaking field } & 14\end{array}$

\section{Introduction}

It is well known that group theoretical properties and number of Nambu-Goldstone (NG) bosons are determined by a given coset space $G / H$. If we extend it to a supersymmetric (SUSY) theory, the NG bosons are always accompanied by fermions. It is extremely interesting to identify the fermions (called as quasi NG fermions) with observed quarks and leptons $[1,2]$, since it may provide us not only an origin of families of quarks and leptons but also an answer to the fundamental question why we have three families in nature.

The SUSY $E_{7}$ non-linear sigma (NLS) model based on the coset space $E_{7} / \mathrm{SU}(5) \times$ $\mathrm{U}(1)^{3}[3,4]$ is fascinating, since it can accommodate three families of quarks and sleptons as its NG chiral multiplets. This suggests that we may predict the maximal number of families in the approach of NLS based on exceptional groups, since the exceptional group is limited up to $E_{8}$. In fact, it is shown that number of the families is limited to be three even if we take the biggest exceptional group $E_{8}[5]$.

In this paper we consider the $E_{7} / \mathrm{SU}(5) \times \mathrm{U}(1)^{3}$ model, where the unbroken $\mathrm{SU}(5)$ is identified with the gauge group of the grand unified theory (GUT). The masses of the squarks and sleptons are highly suppressed at the tree level, since the global $E_{7}$ is assumed to be exact in the limit where gauge couplings and Yukawa couplings vanish. Their masses are dominantly generated via radiative corrections, leading to a natural solution to the flavor changing neutral current (FCNC) problem. On the other hand, Higgs multiplets, $H_{u}$ and $H_{d}$, are considered to be non-NG multiplets, and hence, their masses are not suppressed.

The purpose of this paper is to examine if the above NG boson hypothesis of all the squarks and sleptons is consistent with observations. For this purpose, we choose one of 
the attractive and consistent mediation scenarios of SUSY breaking, that is called as pure gravity mediation (PGM) [6, 7] or minimal split SUSY [8]. In the PGM, gaugino masses are generated radiatively at the one-loop level via anomaly mediation $[9,10]$. Since the gravitino mass is larger than $\mathcal{O}(100) \mathrm{TeV}$, the cosmological gravitino problem is avoided easily. The gaugino masses are generated without a gauge singlet SUSY breaking (Polonyi) field; therefore, the cosmological Polonyi problem does not exist. Even without the gauge singlet SUSY breaking field, the Higgsino mass term of the order of the gravitino mass arises via an $R$-symmetry breaking term, i.e. the constant term in the superpotential $[6,11]$. In the PGM, the lightest neutralino is the pure wino of a mass range of $\mathcal{O}(100-1000) \mathrm{GeV}$, providing us with a good dark matter candidate. Furthermore, it is expected that the SUSY FCNC problem is significantly relaxed.

In this paper, we point out that the $E_{7} / \mathrm{SU}(5) \times \mathrm{U}(1)^{3} \mathrm{NLS}$ model is consistent with pure gravity mediation. ${ }^{1}$ The present model can be regarded as an ultraviolet completion of Higgs-Anomaly mediation proposed in ref. [13]. Furthermore, we also show the predicted squark masses of the first and second generations are likely to be smaller than a few $\mathrm{TeV}$ when the thermal leptogenesis [14] (see also $[15,16]$ for reviews) successfully explains the observed baryon asymmetry. Those squarks can be discovered at the LHC Run-2 or at the high luminosity LHC.

This paper is organized as follows. In section 2, we explain our setup, the $E_{7}$ NLS model combined with PGM. It is shown that the NG multiplets of the three chiral generations have vanishing masses at the tree level. In section 3, we investigate low-energy phenomenology of the model, paying attention to the the lightest squark and slepton masses. Section 4 is devoted to the conclusion.

\section{$2 \quad E_{7} / \mathrm{SU}(5) \times \mathrm{U}(1)^{3}$ model in pure gravity mediation}

The $E_{7} / \mathrm{SU}(5) \times \mathrm{U}(1)^{3}$ model is obtained via $E_{7} / \mathrm{SU}(5) \times \mathrm{SU}(3) \times \mathrm{U}(1)$ model, which contains $133-24-8-1=100 \mathrm{NG}$ modes. The NG multiplets are [3]

$$
\phi_{a}^{i}:(\overline{\mathbf{5}}, \mathbf{3}, 2), \phi_{i}^{a b}:(\mathbf{1 0}, \overline{\mathbf{3}}, 1), \phi^{a}:(\mathbf{5}, \mathbf{1}, 3),
$$

where $a, b=1 \ldots 5$ and $i=1 \ldots 3$. Note that $\phi_{a}^{i}$ and $\phi_{i}^{a b}$ are identified with chiral multiplets of three family leptons and quarks, whose scalar components are massless at the tree level. Here, we assume $\phi^{a}$ has a large Dirac mass term with another $\overline{\mathbf{5}}^{\prime},{ }^{2}$ which is required to cancel the non-linear sigma model and gauge anomalies [4]. The Higgs chiral multiplets, $H_{u}$ and $H_{d}$, are introduced as non-NG matter multiplets. Then, their boson components are expected to have SUSY breaking soft masses of the order of the gravitino mass, $m_{3 / 2}$. The $E_{7} / \mathrm{SU}(5) \times \mathrm{U}(1)^{3}$ model is obtained through the further breaking $\mathrm{SU}(3)$ down to $\mathrm{U}(1)^{2}$, leading to six more NG bosons. These NG bosons are identified with scalar partners of three

\footnotetext{
${ }^{1}$ In ref. [12], it has been shown that gaugino-Higgs mediation is consistent with the $E_{7} / \mathrm{SU}(5) \times \mathrm{U}(1)^{3}$ model. In this case, the higgsino-like neutralino rather than the stau can be the lightest SUSY particle, reducing the fine-tuning of the electroweak symmetry breaking.

${ }^{2}$ We do not identify the NG multiplet $\phi^{a}$ with one of Higgs multiplets, $H_{u}$, in this paper.
} 
right-handed neutrinos. After all, the three new NG chiral multiplets of $E_{7} / \mathrm{SU}(5) \times \mathrm{U}(1)^{3}$ are identified with three chiral multiplets of the right-handed neutrinos.

The Kähler potential for the NG multiplets is constructed from a real function transforming under $E_{7}$ as

$$
\mathcal{K}\left(\phi, \phi^{\dagger}\right) \rightarrow \mathcal{K}\left(\phi, \phi^{\dagger}\right)+f_{H}(\phi)+f_{H}(\phi)^{\dagger},
$$

where $f_{H}$ is a holomorphic function of $\phi$, and $\mathcal{K}$ is invariant under transformations of the unbroken symmetry, $\mathrm{SU}(5) \times \mathrm{U}(1)^{3}$. The real function $\mathcal{K}$ itself can not be $E_{7}$ invariant, since the shift of $\mathcal{K}$ with the holomorphic function does not leave the Lagrangian invariant in supergravity theories: we need an chiral superfield, $S$, to cancel the shift $[17,18]$. Then, we have the $E_{7}$ invariant Kähler potential as

$$
K\left(\phi, \phi^{\dagger}, S, S^{\dagger}\right)=F\left(\mathcal{K}\left(\phi, \phi^{\dagger}\right)+S+S^{\dagger}\right)
$$

with

$$
S \rightarrow S-f_{H}(\phi)
$$

under $E_{7}$ transformation.

Soft masses. To examine a soft SUSY breaking mass for $\phi$, let us consider the leading term of $\phi^{\dagger} \phi$ as

$$
K=F\left(\phi^{\dagger} \phi+S+S^{\dagger}+\ldots\right)
$$

where ... denotes irrelevant terms such as higher order terms of $\phi^{\dagger} \phi$.

The soft SUSY breaking mass of $\phi$ arises from

$$
\begin{aligned}
V\left(S, S^{\dagger}, \phi, \phi^{\dagger}\right) & =e^{K / M_{P}^{2}}\left[\left|\frac{\partial K}{\partial \phi}\right|^{2} K_{\phi \phi}^{-1}+\left|\frac{\partial K}{\partial S}\right|^{2} K_{S S}^{-1}+\frac{\partial K}{\partial \phi} \frac{\partial K}{\partial S^{\dagger}} K_{\phi S}^{-1}+h . c .\right] \frac{|W|^{2}}{M_{P}^{4}} \\
& =e^{K / M_{P}^{2}}\left(\frac{\partial K}{\partial x}\right)^{2}\left(\frac{\partial^{2} K}{\partial x^{2}}\right)^{-1} \frac{|W|^{2}}{M_{P}^{4}}=G(x),
\end{aligned}
$$

where $x=\phi^{\dagger} \phi+S+S^{\dagger} ; W$ is the superpotential with $e^{K / M_{P}^{2}}|W|^{2}=m_{3 / 2}^{2}$. Here, $M_{P} \simeq$ $2.4 \times 10^{18} \mathrm{GeV}$ is the reduced Planck mass. Then,

$$
\frac{\partial^{2} V}{\partial \phi^{\dagger} \partial \phi}=\frac{\partial V}{\partial x}+|\phi|^{2} \frac{\partial^{2} V}{\partial x^{2}}
$$

which vanishes at the minimum. We see all NG bosons are massless at the tree level. ${ }^{3}$ The above argument does not depend on whether there is a direct coupling between a (pseudo) NG multiplet and a SUSY breaking field $Z$, since the potential is just replaced as $G(x) \rightarrow G\left(x, Z, Z^{\dagger}\right)$ (see appendix for an explicit calculation).

\footnotetext{
${ }^{3}$ See also ref. [19] for another clarification of the masslessness.
} 
On the other hand, we assume that the soft SUSY breaking masses for the Higgs doublets, which are non-NG multiplets, are given by:

$$
m_{H_{u}}^{2}=m_{H_{d}}^{2} \simeq c_{H} m_{3 / 2}^{2},
$$

at the tree level, where $c_{H}$ is a constant free parameter. The negativeness of $m_{H_{u}}^{2}$ and $m_{H_{d}}^{2}$ is important to solve the tachyonic slepton problem [13] and give large masses for stops [13, 20], enhancing the Higgs boson mass [21-25]. In fact, the soft SUSY breaking masses for NG bosons arise radiatively via anomaly mediation and renormalization group (RG) running effects from $m_{H_{u}}^{2}$ and $m_{H_{d}}^{2}$. At the high energy scale, say, the GUT scale $\left(M_{\mathrm{GUT}}\right)$, the scalar mass of the NG multiplet is written as

$$
\tilde{m}_{\phi}^{2}\left(\mu_{R}=M_{\mathrm{GUT}}\right) \simeq-\frac{1}{4}\left[\frac{\partial \gamma_{\phi}}{\partial g} \beta_{g}+\frac{\partial \gamma_{\phi}}{\partial y} \beta_{y}\right] m_{3 / 2}^{2},
$$

where $m_{3 / 2}$ is a gravitino mass and $\gamma_{\phi}$ is an anomalous dimension defined by $\gamma_{\phi} \equiv$ $\partial \ln Z_{\phi} / \partial \ln \mu_{R}$ with $\mu_{R}$ of a renormalization scale. For $c_{H}=0$ in eq. (2.8), the above relation holds at any scale and the sleptons are tachyonic below the GUT scale due to the positive beta-functions for the $\mathrm{U}(1)_{Y}$ and $\mathrm{SU}(2)_{L}$ gauge couplings. In the next section, we show that this problem can be solved if the Higgs soft mass squares are negative at $M_{\mathrm{GUT}}[13]$.

Since there is no Polonyi field in our setup, the gaugino masses are purely generated from anomaly mediation:

$$
M_{1}=\frac{33}{5} g_{1}^{2} m_{3 / 2}, M_{2}=g_{2}^{2} m_{3 / 2}, M_{3}=-3 g_{3}^{2} m_{3 / 2},
$$

above a SUSY particle mass scale. Here, $M_{1}, M_{2}$ and $M_{3}$ are bino, wino and gluino mass, respectively; $g_{1}, g_{2}$ and $g_{3}$ are gauge coupling constants of $\mathrm{U}(1)_{Y}, \mathrm{SU}(2)_{L}$ and $\mathrm{SU}(3)_{C}$. Below the SUSY particle mass scale, the threshold corrections [26] need to be included. Trilinear couplings are also generated from anomaly mediation and given by

$$
A_{l m n}=\frac{1}{2}\left(\gamma_{l}+\gamma_{m}+\gamma_{n}\right) m_{3 / 2},
$$

with $V \ni A_{l m n} y_{l m n} \phi_{l} \phi_{m} \phi_{n}+$ h.c.

\section{Phenomenological implications}

\subsection{Case of vanishing squark and slepton masses}

We assume, in this subsection, that the gauge and Yukawa interactions are only sources of the explicit breaking of the global $E_{7}$, and hence all squarks and sleptons are massless at the tree level. ${ }^{4}$ The masses of squark and sleptons arise only through anomaly mediation at the high energy (GUT) scale. Thus, sleptons are tachyonic at $M_{\mathrm{GUT}}$ as discussed in the

\footnotetext{
${ }^{4}$ The quantum corrections other than those from anomaly mediation may be suppressed enough, if one consider the sequestering between the SUSY breaking hidden sector and the MSSM sector (see e.g. appendix in ref. [27]).
} 
previous section. However, radiative corrections from the Higgs loops lift up the slepton mass for $c_{H}<0$, solving the tachyonic slepton problem. We estimate the positive contributions to sfermion masses from Higgs loops by taking into account RG running effects. The resultant mass spectrum is hierarchical among generations since Yukawa couplings of Higgs doublets are hierarchical $[13,20]$, and hence we obtain relatively large masses for stops which explains easily the observed Higgs boson mass of $125 \mathrm{GeV}$.

Since the sleptons and squarks are massless at the tree level, this model has four parameters:

$$
m_{3 / 2}, \tan \beta, c_{H}, \operatorname{sign}(\mu),
$$

where $\tan \beta$ is the ratio of the vacuum expectation values of $H_{u}$ to $H_{d}$. Note that from the conditions of the correct electroweak symmetry breaking, the viable range of $\tan \beta$ is fixed to be $\tan \beta \gtrsim 40$ (60) for $\operatorname{sign}(\mu)=+(-)$. This is because one needs large Yukawa couplings of the tau and bottom, $Y_{\tau}$ and $Y_{b}$.

The necessity of the large $\tan \beta$ is explained as follows. From the electroweak symmetry breaking (EWSB) conditions, we have

$$
m_{A}^{2} \simeq\left(m_{H_{u}}^{2}+m_{H_{d}}^{2}+2|\mu|^{2}\right) \simeq\left(m_{H_{d}}^{2}-m_{H_{u}}^{2}\right)\left(1+2 / \tan ^{2} \beta\right),
$$

where $m_{A}$ is a mass of the CP-odd Higgs. Here, we have used $\mu^{2} \simeq-m_{H_{u}}^{2}+\left(m_{H_{d}}^{2}-\right.$ $\left.m_{H_{u}}^{2}\right) / \tan ^{2} \beta$. In order to avoid a tachyonic CP-odd Higgs, $m_{A}^{2}<0, m_{H_{d}}^{2}>m_{H_{u}}^{2}$ should be satisfied at the electroweak scale. This is achieved via the RG running of $m_{H_{d}}^{2}$,

$$
\frac{d m_{H_{d}}^{2}}{d \ln \mu_{R}} \ni \frac{1}{16 \pi^{2}}\left(6 Y_{b}^{2}+2 Y_{\tau}^{2}\right) m_{H_{d}}^{2}
$$

for large $Y_{b}$ and $Y_{\tau}$ (remember that $m_{H_{d}}^{2}$ is negative). For large $\tan \beta \gtrsim 40$ (or 60 for $\mu<0$ ), the above contribution is larger than that for $m_{H_{u}}^{2}$,

$$
\frac{d m_{H_{u}}^{2}}{d \ln \mu_{R}} \ni \frac{1}{16 \pi^{2}}\left(6 Y_{t}^{2}\right) m_{H_{u}}^{2} \text {. }
$$

Then, $m_{H_{d}}^{2}>m_{H_{u}}^{2}\left(\left|m_{H_{d}}^{2}\right|<\left|m_{H_{u}}^{2}\right|\right)$ is realized at the low-energy scale, which is required for the correct EWSB.

The dependence of the required $\tan \beta$ on $\operatorname{sign}(\mu)$ originates from the radiative correction to $Y_{b}$ from sbottom-gluino loops $[28,29]$. Since the SUSY contribution to the muon $g-2$ is opposite to the needed one and the required $\tan \beta$ for correct EWSB is so large in the case of $\operatorname{sign}(\mu)=-$, we focus on the region of $\operatorname{sign}(\mu)=+$ in this paper, unless otherwise noted. In fact, we can not find particular differences in the SUSY-particle spectra between the regions with $\operatorname{sign}(\mu)=-$ and $\operatorname{sign}(\mu)=+$, apart from the required $\tan \beta$ and the contribution to the muon $g-2$.

The SUSY spectrum is computed using SuSpect 2.4.3 [30]. We scan all allowed parameter space of $c_{H}$ and $\tan \beta$,

$$
0>c_{H}>-0.5, \quad 40<\tan \beta<60,
$$



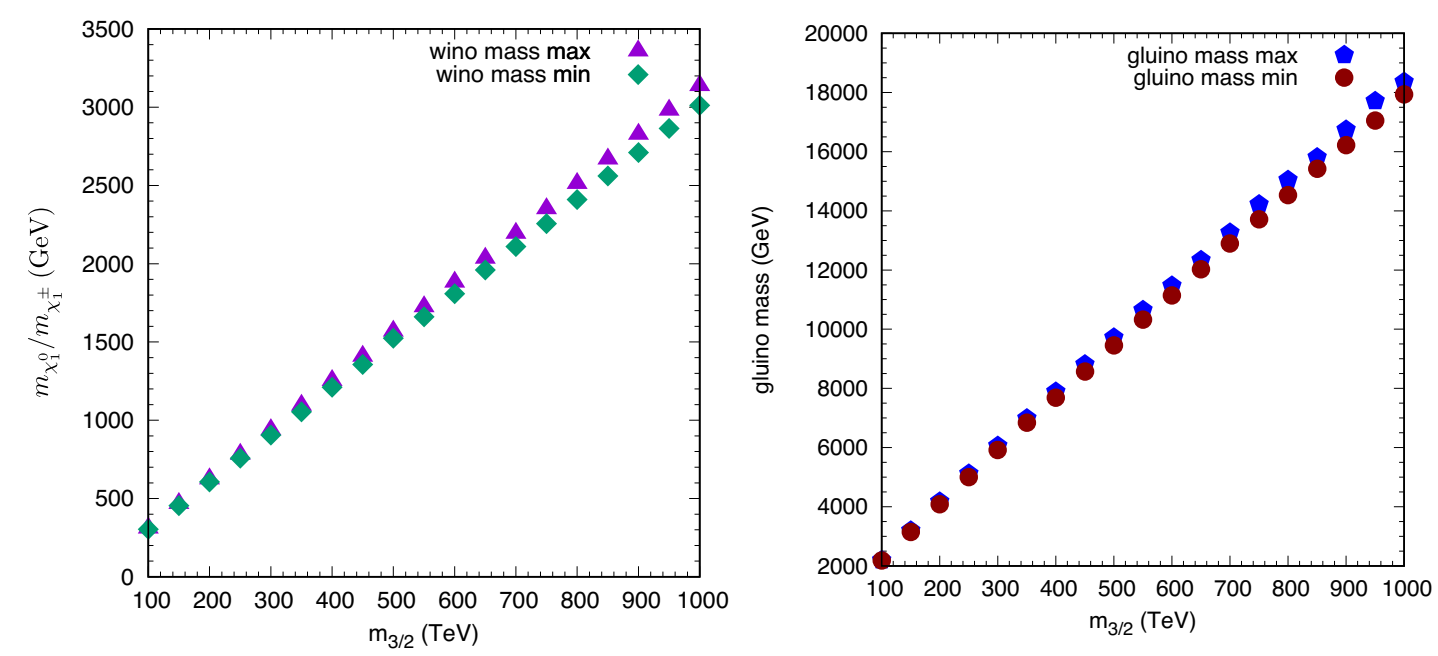

Figure 1. The masses of the lightest chargino/neutralino and the gluino in the case of the vanishing squark and slepton masses. The triangles and pentagons (squares and circles) show maximum (minimum) values. Here, $\alpha_{s}\left(m_{Z}\right)=0.1185$ and $m_{t}($ pole $)=173.34 \mathrm{GeV}$.

and take the maximum and minimum values of $m_{\chi_{1}}^{ \pm} / m_{\chi_{1}^{0}}$ for each $m_{3 / 2}$. Here, $m_{\chi_{1}}^{ \pm}$and $m_{\chi_{1}^{0}}$ are the lightest chargino mass and neutralino mass, respectively, and they are nearly degenerated. The input parameter $c_{H}$ is set at $M_{\mathrm{inp}}=10^{16} \mathrm{GeV}\left(\approx M_{\mathrm{GUT}}\right)$. We have demanded the lightest slepton (squark) to be heavier than 340 (1000) GeV from the null results of the LHC SUSY searches [31, 32]. ${ }^{5}$

The range of $c_{H}$ has been determined to avoid the tachyonic squarks and sleptons: squarks become tachyonic in the regions of $c_{H}<-0.5$. Note that on the viable parameter space, we find that all Yukawa couplings at the GUT scale are smaller than $\sqrt{0.1 \times 4 \pi}$.

We now show the mass of the lightest chargino, which is almost pure wino, in figure 1 (left). We see the wino mass is almost insensitive to $\tan \beta$ and $c_{H}$. This is because the threshold correction [26]

$$
\frac{\Delta M_{2}}{M_{2}} \ni \frac{g_{2}^{2}}{16 \pi^{2}} \frac{\mu}{M_{2}} \sin (2 \beta) \frac{m_{A}^{2}}{\mu^{2}-m_{A}^{2}} \ln \frac{\mu^{2}}{m_{A}^{2}}
$$

is suppressed by large $\tan \beta$ and $\mu^{2} \gg m_{A}^{2}$ : in contrast to usual PGM models, the relation between wino mass and gravitino mass is almost fixed. Since the Higgsino is heavy, the leading dark matter candidate in this model is the wino-like neutralino. This is the reason why we restrict the range of $m_{3 / 2} \simeq 100-1000 \mathrm{TeV}$, (approximately) corresponding to the mass range for the wino dark matter, $270 \mathrm{GeV}<m_{\tilde{\chi}_{1}^{0}}<2.9 \mathrm{TeV}$. This is followed by the phenomenological constraints assuming that the wino is the lightest SUSY particle (LSP). The lower-bound comes from the wino search at the LHC [33] while the upper-bound

\footnotetext{
${ }^{5}$ The lower bound of the slepton mass of $340 \mathrm{GeV}$ is given in the case that the slepton is lighter than the lightest neutralino and effectively stable inside the detectors.
} 


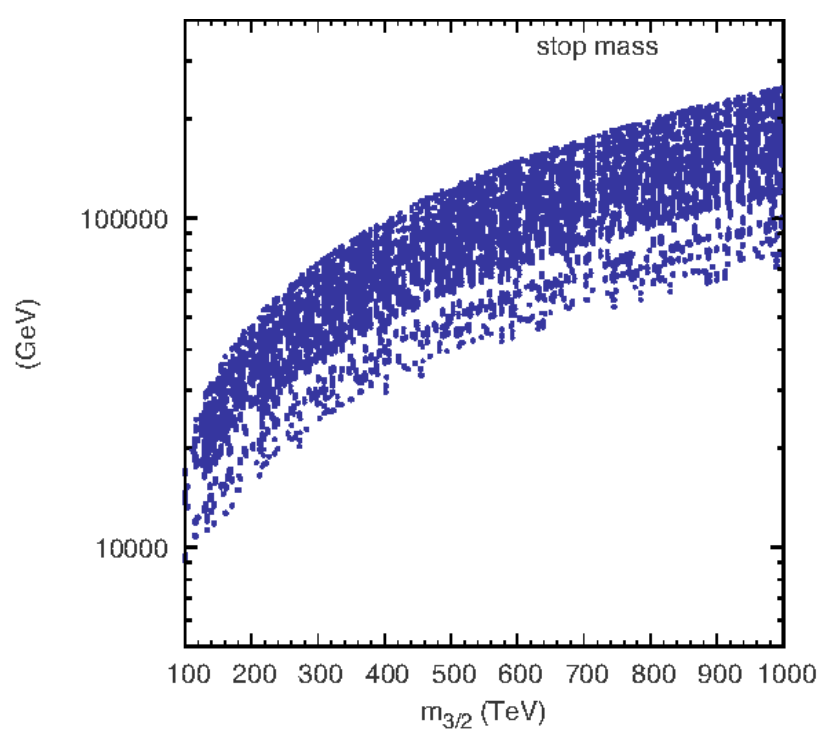

Figure 2. Scatter plot of the stop mass.

is given by the condition to avoid the over-closure of the universe with the wino dark matter [34]. ${ }^{6}$

On the right panel in figure 1, we also show the maximum and minimum values of the gluino mass. The gluino is accessible at the LHC only when the gravitino mass is smaller than about $150 \mathrm{TeV}$.

Next, we show the stop mass, defined by $m_{\tilde{t}} \equiv\left(m_{Q_{3}} m_{\bar{U}_{3}}\right)^{1 / 2}$ in figure 2 to estimate regions consistent with the Higgs boson mass of $125 \mathrm{GeV}$. Here, $m_{Q_{3}}$ and $m_{\bar{U}_{3}}$ are the masses of the left-handed and right-handed stop, respectively. The scanned range of the parameter space is the same as in eq. (3.5). The stop mass is lifted up via RG running:

$$
\frac{d m_{Q_{3}^{2}}}{d \ln \mu_{R}} \ni \frac{1}{16 \pi^{2}}\left(2 Y_{t}^{2} m_{H_{u}}^{2}+2 Y_{b}^{2} m_{H_{d}}^{2}\right), \quad \frac{d m_{\bar{U}_{3}}^{2}}{d \ln \mu_{R}} \ni \frac{1}{16 \pi^{2}} 4 Y_{t}^{2} m_{H_{u}}^{2} .
$$

Therefore, the large $\left|c_{H}\right|$ leads to the larger stop masses. On almost all points, the stop mass is larger than $10 \mathrm{TeV},{ }^{7}$ and hence the Higgs boson mass of $125 \mathrm{GeV}$ is easily explained in most of the parameter space.

In figure 3, the scatter plots of the masses of the lightest squark and slepton are shown. Here, the lightest squark is either the right-handed up squark or down squark, while the lightest slepton is the left-handed or right-handed selectron, depending on the parameter space. The threshold corrections to the squark masses are included utilizing one-loop RG equations (see e.g. [35]) to incorporate the large mass hierarchy between the third generation squarks and first/second generation squarks. ${ }^{8}$ The dark-green and red dots satisfy the constraint, $122 \leq m_{h} \leq 128 \mathrm{GeV}$, while the gray dots do not. Here, $m_{h}$

\footnotetext{
${ }^{6}$ In our case only some of the squarks are light as $1000 \mathrm{GeV}$, i.e. the event cross sections are expected to be smaller than those used in the reference.

${ }^{7}$ Only in the case that $\left|c_{H}\right|$ is as small as 0.04 and $m_{3 / 2}=100 \mathrm{TeV}$, the stop mass becomes $\sim 9 \mathrm{TeV}$.

${ }^{8}$ We have modified the SuSpect code to include the resummation of the logarithmic corrections.
} 

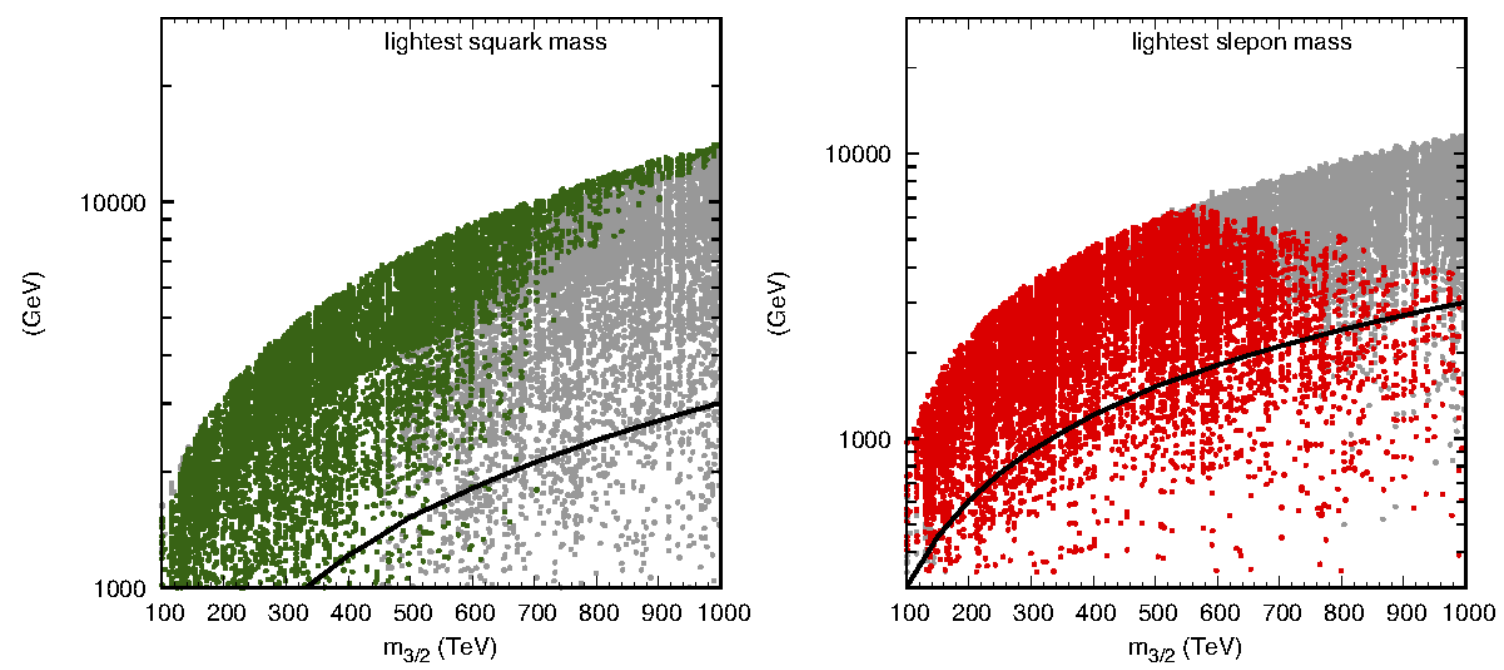

Figure 3. Scatter plots of the lightest squark mass (left) and the lightest slepton mass (right). The dark-green and red dots satisfy the constraint $122 \mathrm{GeV} \leq m_{h} \leq 128 \mathrm{GeV}$ while the gray dots do not. The black solid lines show the minimum values of $m_{\chi_{1}^{0}}$

is the Higgs boson mass computed using SUSYHD 1.0.2 [36]. The black solid lines show the minimum values of $m_{\chi_{1}^{0}}$ in figure 1 . Thus, the points below the lines are excluded unless the $R$-parity is violated. It can be seen that the lightest squark can be as light as $1-2 \mathrm{TeV}$ even for $m_{3 / 2}=1000 \mathrm{TeV}$. However, the stop mass is too large in such cases and $m_{h}$ becomes larger than $128 \mathrm{GeV}$ : larger $\left|c_{H}\right|$ leads to smaller (larger) squark (stop) masses. Considering the Higgs boson mass constraint and the sizes of the squark mass and the lightest neutralino mass, it is expected that the regions for $m_{3 / 2} \lesssim 300-350 \mathrm{TeV}$ can be tested at the high-luminosity LHC [37]. ${ }^{9}$

Regions favored by the thermal leptogenesis. If the thermal leptogenesis is responsible for the observed baryon asymmetry, the mass of the wino-like neutralino needs to be smaller than about $1 \mathrm{TeV}[7]$ when it is the stable DM. This is because the neutralino produced from the gravitino decay leads to the over-closure of the universe for $m_{\chi_{1}^{0}} \gtrsim 1 \mathrm{TeV}$, if the reheating temperature is higher than $\sim 10^{9} \mathrm{GeV}$ due to the large energy density of the gravitino. Note that the reheating temperature higher than about $\sim 10^{9} \mathrm{GeV}$ is required for the successful thermal leptogenesis [38, 39]. The critical value, $m_{\chi_{1}^{0}} \simeq 1 \mathrm{TeV}$, corresponds to $m_{3 / 2} \simeq 320 \mathrm{TeV}$; therefore the regions with $m_{3 / 2} \sim 300 \mathrm{TeV}$ or smaller are especially interesting.

In figure 4, we show the contours of the lightest squark mass (left) and slepton mass (right) as well as $m_{h}$. On the upper (lower) two panels, we take $m_{3 / 2}=150$ (300) TeV. In the gray regions, the lightest slepton is lighter than $340 \mathrm{GeV}$ or the lightest squark is lighter

\footnotetext{
${ }^{9}$ One may consider the case that the $R$-parity is slightly violated. In this case, the viable region is much wider, since the sleptons and squarks can be lighter than $\chi_{1}^{0}$. The region with $m_{3 / 2} \lesssim 700 \mathrm{TeV}$ may be tested at the LHC by searching an $R$-hadron. Notably, the region for even heavier gravitino may be also tested by searching a stable slepton in both LHC and ILC. (The sleptons can be $\mathcal{O}(100) \mathrm{GeV}$ even for $m_{3 / 2}=1000 \mathrm{TeV}$.)
} 

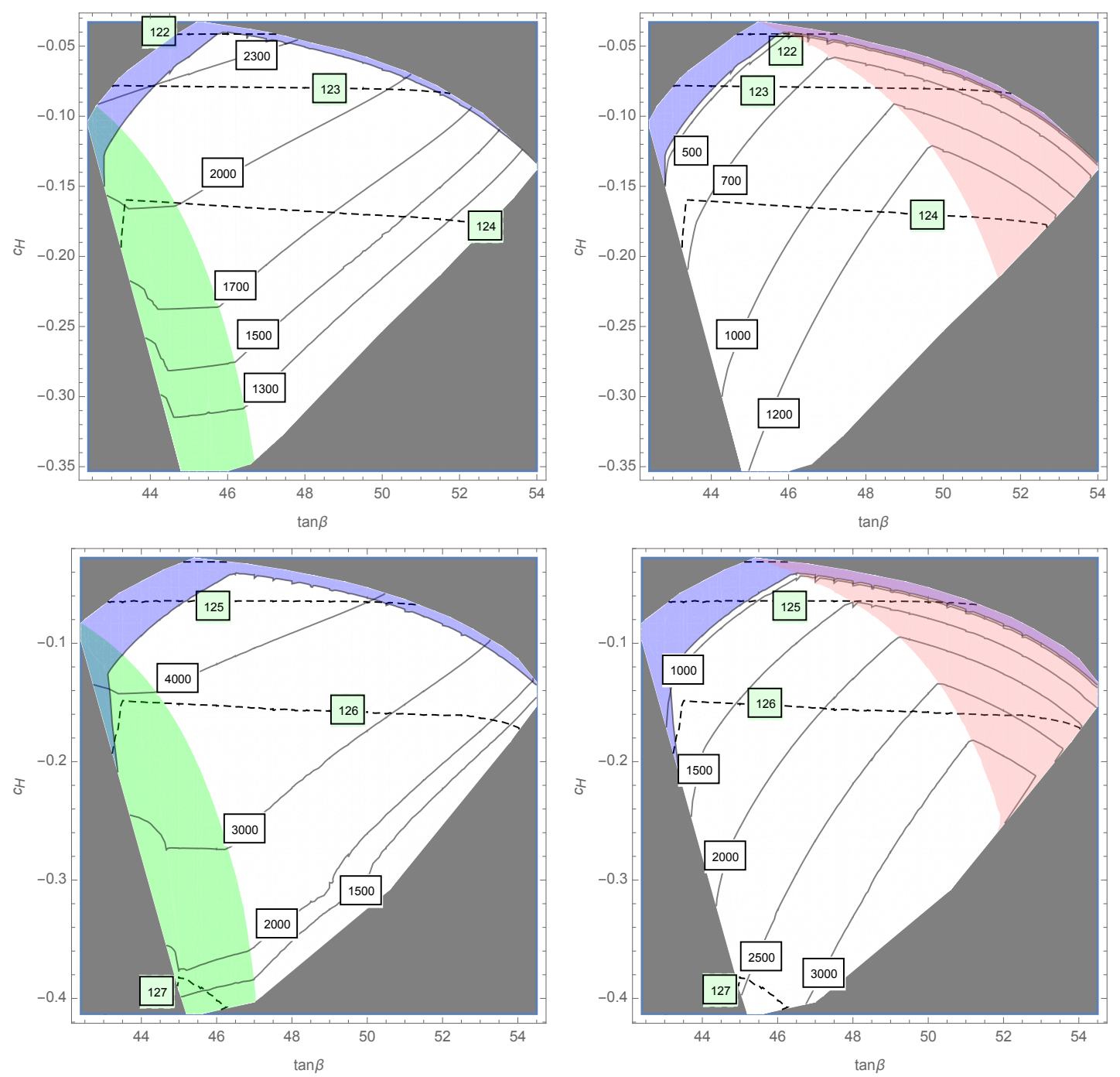

Figure 4. Contours of the lightest squark mass (left panels) and slepton mass (right panels) in unit of GeV. The upper (lower) two panels the gravitino mass is taken as $m_{3 / 2}=150$ (300) TeV. The Higgs boson masses in unit of $\mathrm{GeV}$ are shown as dashed lines. In the blue shaded region, the selectron is the LSP. In the green (pink) shaded regions, the lightest squark (slepton) is the right-handed down squark (the left-handed selectron).

than $1 \mathrm{TeV}$; hence, those regions are likely to be excluded. In the blue shaded region, the slepton is lighter than $\chi_{1}^{0} / \chi_{1}^{ \pm}$and the LSP. In the green (pink) shaded regions, the lightest squark (slepton) is the right-handed down squark (left-handed selectron). [Thus, the righthanded strange squark (the left-handed smuon) is also light.] In the other regions of the left (right) panels, the right-handed up squark (right-handed selectron) is the lightest squark (slepton). (The right-handed smuon is also light.) In the wide regions, the lightest squark is lighter than $3 \mathrm{TeV}$, which may be discovered at the future LHC experiments. Also, the slepton can be light as $500 \mathrm{GeV}$ for $m_{3 / 2}=150 \mathrm{TeV}$. 

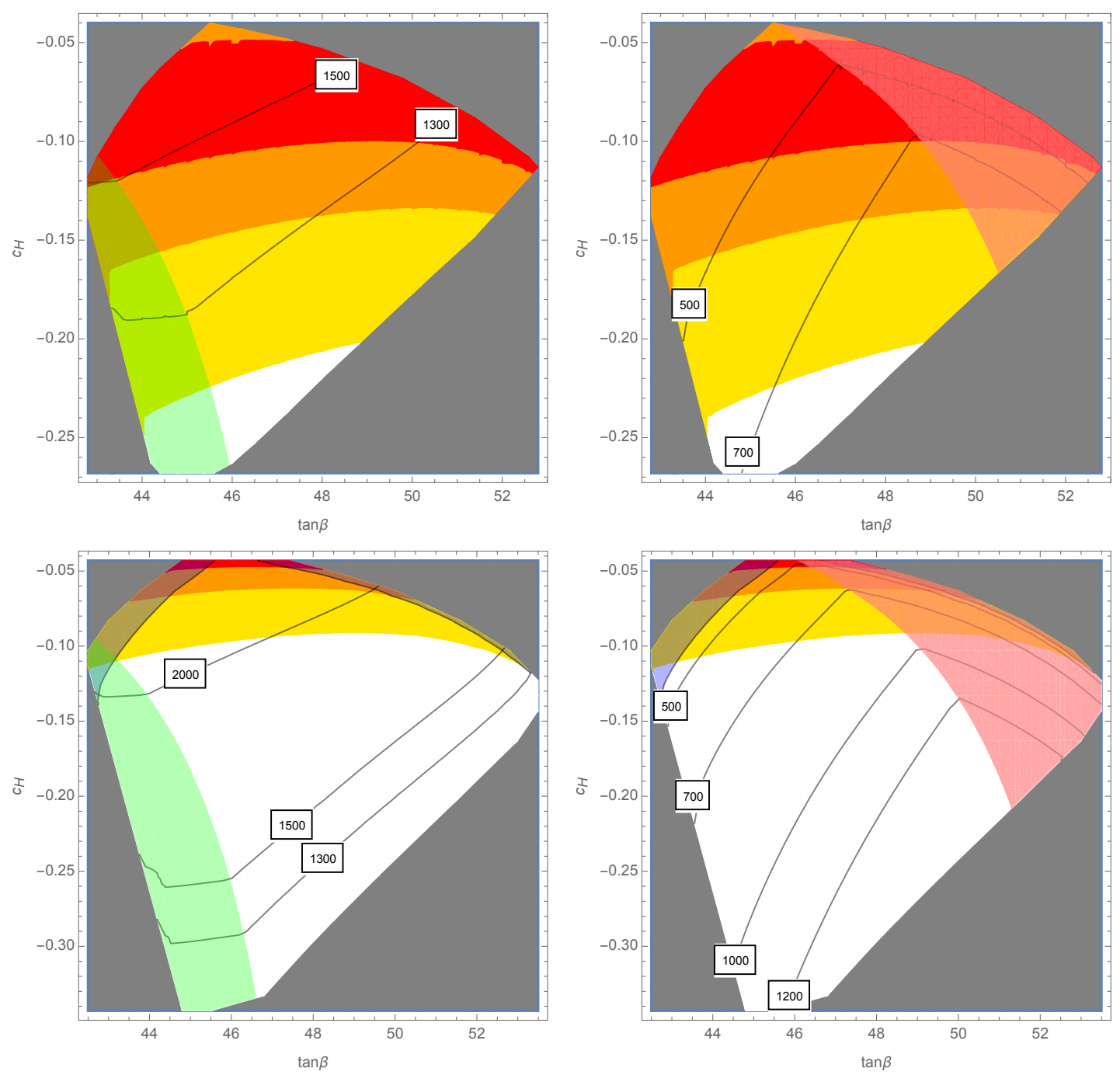

Figure 5. The regions consistent with the muon $g-2$ experiment at $1 \sigma$ (red), $1.5 \sigma$ (orange) and $2 \sigma$ (yellow) level. In the upper two panels, the gravitino mass is taken as $m_{3 / 2}=100(140) \mathrm{TeV}$. We also show the contours of the lightest squark (slepton) mass in the left (right) panels, in unit of $\mathrm{GeV}$. In the blue shaded regions, the selectron is the LSP.

Muon $\boldsymbol{g}-\mathbf{2}$. Finally, let us briefly comment on regions where the muon $g-2$ anomaly $[40-42]^{10}$ is explained via SUSY contributions [44-46]. Since the sleptons and bino can be light as $\mathcal{O}(100) \mathrm{GeV}$ and $\simeq 1 \mathrm{TeV}$, respectively for $m_{3 / 2} \sim 100 \mathrm{TeV}$ and $\mu \tan \beta$ is as large as $\sim \mathcal{O}\left(10^{3}\right) \mathrm{TeV}$, there are regions consistent with the muon $g-2$ experiment. In figure 5 , we show the regions consistent with the experimental value of the muon $g-2$ at $1 \sigma$ (red), $1.5 \sigma$ (orange) and $2 \sigma$ (yellow) level. In the left (right) panels, the contours of the lightest squark (slepton) mass are also shown. We take $m_{3 / 2}=100(140) \mathrm{TeV}$ in the upper (lower) two panels. We include leading two-loop corrections to the muon $g-2$ : the correction to the muon Yukawa coupling [47] and the logarithmic QED correction [48].

\footnotetext{
${ }^{10}$ See also [43] for a standard model prediction of the muon $g-2$.
} 

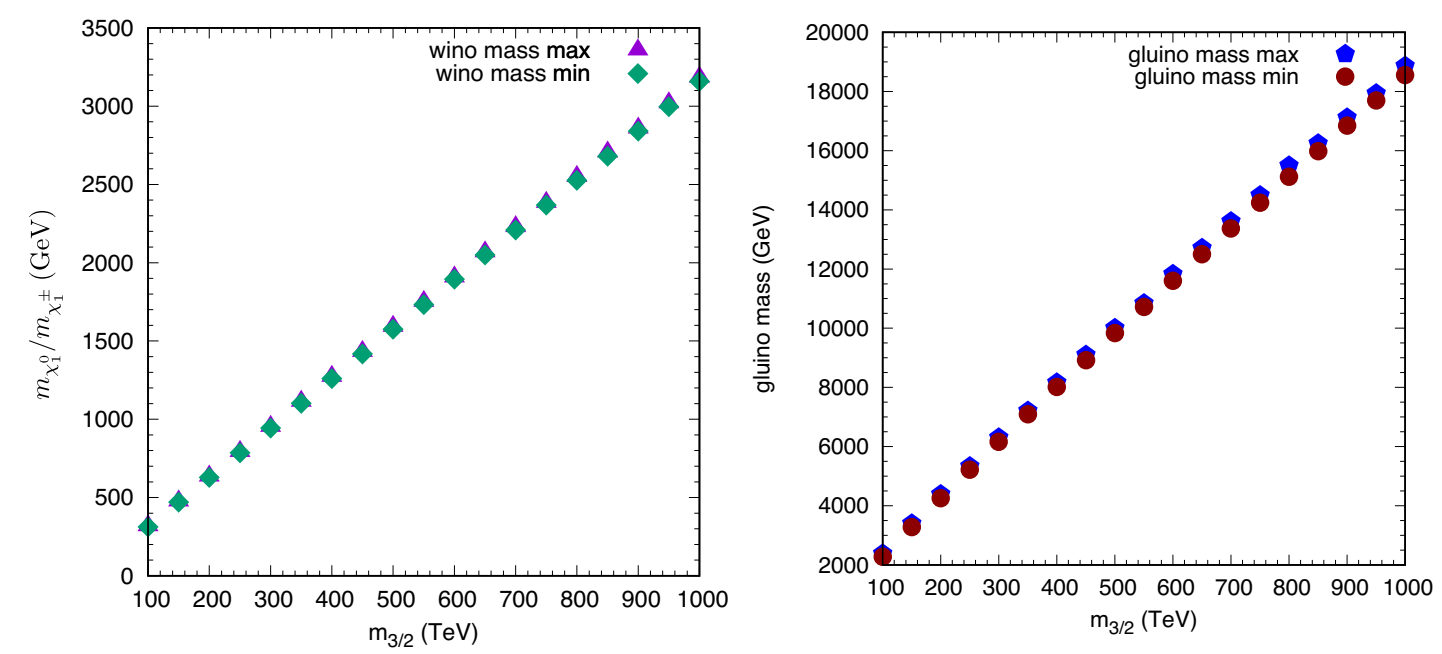

Figure 6. The masses of the lightest chargino/neutralino and the gluino with $m_{0}$. The triangles and pentagons (squares and circles) show maximum (minimum) values.

These regions are explored in details in ref. [13], where further information such as the bottom-tau Yukawa unification and bottom-tau-top Yukawa unification can be found. ${ }^{11}$ Note that in the regions consistent with the muon $g-2$ at $1 \sigma$ level, the wino mass is less than about $500 \mathrm{GeV}$; therefore, these regions are expected to be covered at the high luminosity running of the LHC.

\subsection{Case of non-vanishing squark and slepton masses}

Since Yukawa and gauge couplings break $E_{7}$ explicitly, there might be small SUSY-breaking soft masses for the squarks and sleptons. In this subsection, we consider the case that squark and sleptons have a common soft mass $m_{0}$ of $\mathcal{O}(1) \mathrm{TeV}$ in addition to the contribution from anomaly mediation in eq. (2.9). Notice that $m_{0}$ breaks the $E_{7}$ symmetry explicitly. The model parameters in this setup are

$$
m_{0}, m_{3 / 2}, \tan \beta, c_{H}, \operatorname{sign}(\mu)
$$

The masses of the lightest chargino and the gluino are shown in figure 6 . We take $\operatorname{sign}(\mu)=+$. The maximum (minimum) values are denoted as triangles and pentagons (squares and circles). The lightest chargino (neutralino) is almost pure wino as in the case of section 3.1. We scan the parameter space over the ranges

$$
40<\tan \beta<60, \quad 1000 \mathrm{GeV}+m_{3 / 2} / 100<m_{0}<2\left(1000 \mathrm{GeV}+m_{3 / 2} / 100\right),
$$

with the fixed $c_{H}, c_{H}=-1$. The result is very similar to the one in figure 1 , where there is an approximate one-to-one correspondence between the chargino mass and the gravitino mass.

\footnotetext{
${ }^{11}$ SUSY models beyond the MSSM explaining the muon $g-2$ are shown in refs. [49-55].
} 

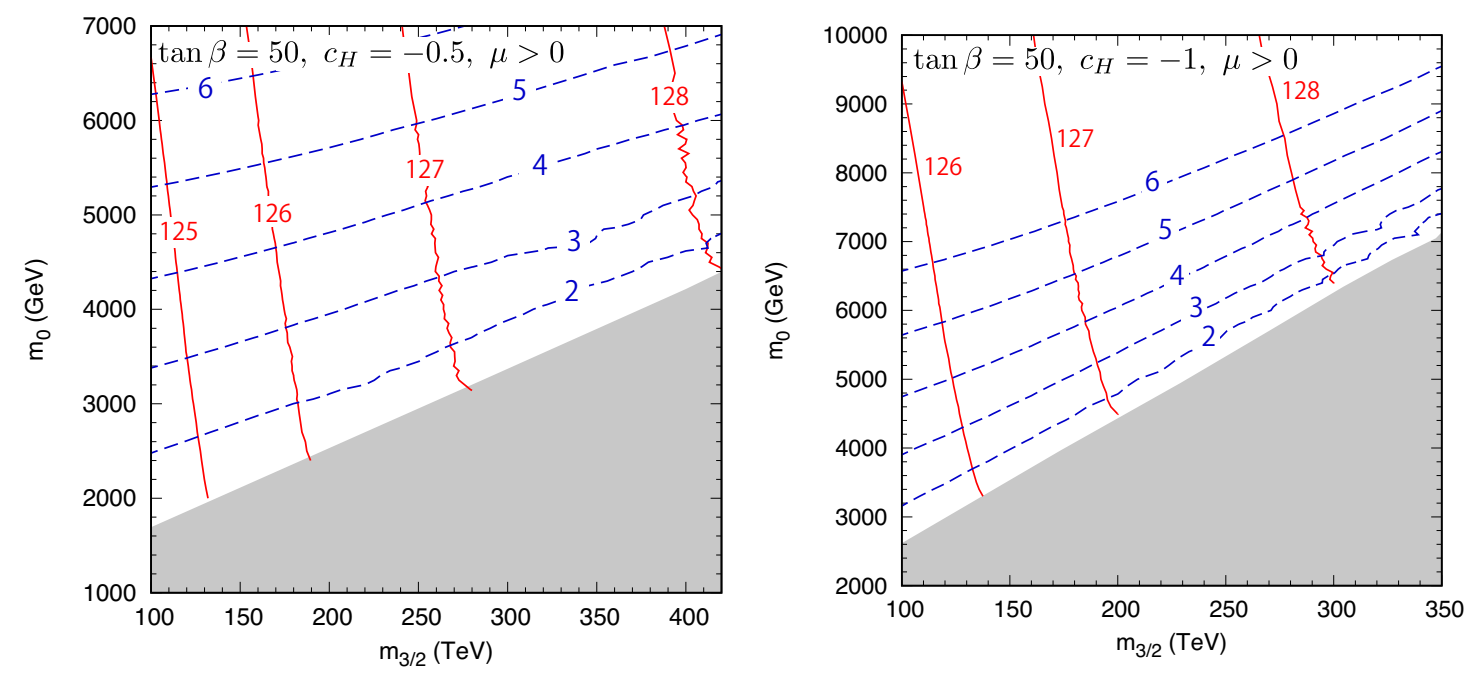

Figure 7. The contours of $m_{h}(\mathrm{GeV})$ and $m_{\tilde{q}}(\mathrm{TeV})$ for $c_{H}=-0.5$ and -1 . The red solid (blue dashed) line shows $m_{h}\left(m_{\tilde{q}}\right)$. Here, $m_{\tilde{q}}$ is a mass of the lightest squark.

In figure 7 , we show the contours of the squark mass and $m_{h}$ on $m_{3 / 2}-m_{0}$ plane for $c_{H}=-0.5$ (left) and -1 (right). We set $\tan \beta=50$. We focus on the viable parameter space, where $122 \leq m_{h} \leq 128$, corresponding to the gravitino mass up to 400 (300) TeV for $c_{H}=-0.5(1)$. If the universal scalar mass at $M_{\text {inp }}$ is smaller than $3-4 \mathrm{TeV}$, the lightest squark is lighter than about $3.5 \mathrm{TeV}$, which may be accessible to the future LHC experiments.

\subsection{Mass spectra}

We show mass spectra at some example points in the viable parameter space of the model (table 1). On the points I-III, the masses of the squarks and sleptons vanish at $M_{\text {inp }}$, while on the point IV, we introduce the small universal mass $m_{0}$. On the point III, $\operatorname{sign}(\mu)=-$; therefore, $\tan \beta$ is large as $\sim 60$. On the point II, the smuons are light and the SUSY contribution to the muon $g-2$ is $1.4 \times 10^{-9}$. On that point, the calculated Higgs boson mass using SUSYHD is $\simeq 122 \mathrm{GeV}$. However, the stop mass is as large as $15 \mathrm{TeV}$, and the computed Higgs mass using FeynHiggs 2.12 .0 [56-60] is larger than $125 \mathrm{GeV}$; therefore, the point could be consistent with the observed Higgs boson mass. Although $\mu \tan \beta$ is very large in our scenario and there may exist a charge breaking minimum deeper than the EWSB one in the smuon-Higgs potential, the constraint from the (meta) stability of the EWSB minimum is not severe due to the small Yukawa coupling of the muon [61]. On the listed points, the mass of the lightest squark is smaller than $3 \mathrm{TeV}$, which may be tested at the LHC Run-2 or at the high luminosity LHC. 


\begin{tabular}{|c||c|c|c|c|}
\hline Parameters & Point I & Point II & Point III & Point IV \\
\hline$m_{3 / 2}(\mathrm{TeV})$ & 320 & 140 & 250 & 100 \\
$c_{H}$ & -0.25 & -0.06 & -0.2 & -1 \\
$\tan \beta$ & 50 & 47 & 62 & 50 \\
$m_{0}(\mathrm{GeV})$ & 0 & 0 & 0 & 3000 \\
$\operatorname{sign}(\mu)$ & + & + & - & + \\
\hline Particles & Mass $(\mathrm{GeV})$ & Mass $(\mathrm{GeV})$ & Mass $(\mathrm{GeV})$ & Mass $(\mathrm{GeV})$ \\
\hline$\tilde{g}$ & 6350 & 2960 & 5060 & 2330 \\
$\tilde{q}$ & $2340-5470$ & $2130-2410$ & $2990-4430$ & $1760-3490$ \\
$\tilde{t}_{2,1}(\mathrm{TeV})$ & 62,62 & 15,14 & 44,41 & 39,37 \\
$\tilde{b}_{2,1}(\mathrm{TeV})$ & 65,64 & 16,15 & 42,39 & 39,38 \\
$\tilde{\chi}_{1}^{0} / \tilde{\chi}_{1}^{ \pm}$ & 993 & 441 & 790 & 323 \\
$\tilde{\chi}_{2}^{0}$ & 2990 & 1290 & 2340 & 934 \\
$\tilde{e}_{L, R}$ & 4200,3310 & 700,662 & 3150,1720 & 4230,3530 \\
$\tilde{\mu}_{L, R}$ & 4480,3980 & 757,779 & 4530,4900 & 4420,3980 \\
$\tilde{\tau}_{2,1}(\mathrm{TeV})$ & 60,43 & $13,8.9$ & 52,37 & 38,27 \\
$H^{ \pm}(\mathrm{TeV})$ & 47 & 12 & 25 & 22 \\
$h_{\mathrm{SM}-\mathrm{like}}$ & 126.6 & 122.3 & 125.5 & 125.2 \\
\hline$\mu(\mathrm{TeV})$ & 140 & 30 & -98 & 88 \\
\hline \multicolumn{4}{|r|}{}
\end{tabular}

Table 1. Mass spectra in sample points.

\section{Conclusion}

The $E_{7} / \mathrm{SU}(5) \times \mathrm{U}(1)^{3}$ non-linear sigma model is very attractive, since it may provide us an intriguing answer to one of the fundamental questions, why we have three families of quarks and leptons. In the $E_{7}$ NLS model, the masses of squarks and sleptons vanish at the tree level while the Higgs doublets have soft SUSY breaking masses of the order of the gravitino mass.

In this paper, we have shown that the $E_{7} / \mathrm{SU}(5) \times \mathrm{U}(1)^{3} \mathrm{NLS}$ model is consistent with all observations if one adopts the pure gravity mediation or minimal split SUSY, where the gaugino masses arise only from anomaly mediation. The tachyonic slepton problem in anomaly mediation is solved due to the renormalization group running effects from the negative Higgs soft mass squares. We have shown that if the observed baryon asymmetry is explained by the thermal leptogenesis, the squarks are lighter than $2-3 \mathrm{TeV}$ in a wide range of the viable parameter space, and we expect them to be discovered at the LHC Run-2 or at the high luminosity running. Moreover, the sleptons may be as light as $\mathrm{O}(100) \mathrm{GeV}$, giving rise to a possibility for explaining the muon $g-2$ anomaly. The sleptons are also interesting target at the LHC and at ILC.

Although we have concentrated on the case that the $R$-parity is conserved, one can also consider the small $R$-parity violation. In this case, it is easy to imagine that the viable parameter region becomes wider, since the squarks and sleptons can be lighter than the 
lightest neutralino. Note that the testability of the model is also enhanced: the stable squark can be checked by searching an $R$-hadron for heavy gravitino of $\sim 700 \mathrm{TeV}$; the stable slepton can be as light as $\mathcal{O}(100) \mathrm{GeV}$ even for $m_{3 / 2}=1000 \mathrm{TeV}$.

Finally, let us comment on the SUSY CP-problem for $m_{3 / 2}=100-300 \mathrm{TeV}$, where the selectron, bino and first/second generation squarks are as light as a few $\mathrm{TeV}$. In this case, the constraints from the electron and neutron electric dipole moment (EDM) are severe. For instance, in the base that the $\mu$-term and gravitino mass are real, the argument of the Higgs $B$-term needs to be as small as $10^{-3}-10^{-4}$ to avoid the constraint from the electron EDM [62]. It is, however, remarkable that we need CP violation only in the $E_{7}$-breaking Yukawa couplings, so far. Thus, it is very interesting to consider that violations of two independent symmetries, $\mathrm{CP}$ and $E_{7}$, arise from an underlying common physics.

\section{Acknowledgments}

This work is supported by JSPS KAKENHI Grant Numbers JP26104009 (T.T.Y), JP26287039 (T.T.Y.), JP16H02176 (T.T.Y), JP15H05889 (N.Y.) and JP15K21733 (N.Y.); and by World Premier International Research Center Initiative (WPI Initiative), MEXT, Japan (T.T.Y.).

\section{A Vanishing soft mass for NG multiplet with direct coupling to the SUSY breaking field}

To explicitly see that the soft mass of the NG multiplet vanishes even if there is a direct coupling to the SUSY breaking field, let us consider the following Kähler potential and superpotential:

$$
K=f(x)\left(1+c Z^{\dagger} Z\right)+Z^{\dagger} Z, \quad W=W(Z)+\mathcal{C},
$$

where $x=\phi^{\dagger} \phi+S+S^{\dagger}$ and $\mathcal{C}$ is a constant term; $S$ is required for the $E_{7}$ invariance of the Lagrangian. We take unit of $M_{P}=1$.

$$
\begin{aligned}
K_{\phi \phi} & =\left(f^{\prime}+|\phi|^{2} f^{\prime \prime}\right)\left(1+c|Z|^{2}\right) \\
K_{S S} & =f^{\prime \prime}\left(1+c|Z|^{2}\right) \\
K_{Z Z} & =1+c f \\
K_{S \phi} & =\phi^{\dagger} f^{\prime \prime}\left(1+c|Z|^{2}\right) \\
K_{Z \phi} & =\phi^{\dagger} f^{\prime}(c Z) \\
K_{Z S} & =f^{\prime}(c Z),
\end{aligned}
$$

where $K_{i j}=\frac{\partial^{2} K}{\partial q_{i}^{\dagger} q_{j}}$. We see that

$$
\begin{aligned}
\operatorname{det}(K) & =-\left(1+c|Z|^{2}\right) f^{\prime}\left[c^{2}|Z|^{2} f^{2}-\left(1+c|Z|^{2}\right)(1+c f) f^{\prime \prime}\right] \\
& =F\left(x,|Z|^{2}\right)
\end{aligned}
$$

where the dependence on $|\phi|^{2}$ arises only though $x$. 
First consider the simple case: $\langle Z\rangle=0$,

$$
\begin{aligned}
K_{\phi \phi} & =f^{\prime}+|\phi|^{2} f^{\prime \prime}, \\
K_{S S} & =f^{\prime \prime} \\
K_{Z Z} & =1+c f, \\
K_{S \phi} & =\phi^{\dagger} f^{\prime \prime}, \\
K_{Z \phi} & =0 \\
K_{Z S} & =0 .
\end{aligned}
$$

The inverse matrix is almost same as in the case of section 2; thus, the argument in section 2 is still valid, even if there is an additional term:

$$
V \ni e^{K}\left|\frac{\partial W}{\partial Z}\right|^{2} \frac{1}{1+c f}=G_{1}\left(x,|Z|^{2}\right) .
$$

So far, $\frac{\partial^{2} V}{\partial \phi^{\dagger} \partial \phi}$ vanishes at the minimum.

Next we consider the case $\langle Z\rangle \neq 0$. The inverse matrix is given by

$$
\begin{aligned}
\operatorname{det}(K) K_{\phi \phi}^{-1} & =-c^{2}|Z|^{2} f^{\prime 2}+\left(1+c|Z|^{2}\right)(1+c f) f^{\prime \prime}, \\
\operatorname{det}(K) K_{S S}^{-1} & =-c^{2}|Z|^{2}\left|\phi^{2}\right| f^{\prime 2}+\left(1+c|Z|^{2}\right)(1+c f)\left(f^{\prime}+|\phi|^{2} f^{\prime \prime}\right), \\
\operatorname{det}(K) K_{Z Z}^{-1} & =\left(1+c|Z|^{2}\right) f^{\prime} f^{\prime \prime} \\
\operatorname{det}(K) K_{\phi S}^{-1} & =\phi\left[c^{2}|Z|^{2} f^{\prime 2}-\left(1+c|Z|^{2}\right)(1+c f) f^{\prime \prime}\right] \\
\operatorname{det}(K) K_{\phi Z}^{-1} & =0 \\
\operatorname{det}(K) K_{S Z}^{-1} & =-c\left(1+c|Z|^{2}\right) Z^{\dagger} f^{\prime 2}
\end{aligned}
$$

Therefore, only

$$
V \ni e^{K}|W|^{2}\left[\left|\frac{\partial K}{\partial \phi}\right|^{2} K_{\phi \phi}^{-1}+\left|\frac{\partial K}{\partial S}\right|^{2} K_{S S}^{-1}+\frac{\partial K}{\partial S} \frac{\partial K}{\partial \phi^{\dagger}} K_{S \phi}^{-1}+h . c .\right]=G_{2}\left(x,|Z|^{2}\right)
$$

is relevant as in eq. (2.6). This $G_{2}$ gives the vanishing soft mass to the NG multiplet.

Open Access. This article is distributed under the terms of the Creative Commons Attribution License (CC-BY 4.0), which permits any use, distribution and reproduction in any medium, provided the original author(s) and source are credited.

\section{References}

[1] W. Buchmüller, S.T. Love, R.D. Peccei and T. Yanagida, Quasi Goldstone Fermions, Phys. Lett. B 115 (1982) 233 [INSPIRE].

[2] W. Buchmüller, R.D. Peccei and T. Yanagida, Quarks and Leptons as Quasi Nambu-Goldstone Fermions, Phys. Lett. B 124 (1983) 67 [InSPIRE].

[3] T. Kugo and T. Yanagida, Unification of Families Based on a Coset Space E7/SU(5) $\times \mathrm{SU}(3) \times \mathrm{U}(1)$, Phys. Lett. B $134(1984) 313$ [INSPIRE]. 
[4] T. Yanagida and Y. Yasui, Supersymmetric nonlinear $\sigma$-models based on exceptional groups, Nucl. Phys. B 269 (1986) 575 [inSPIRE].

[5] S. Irie and Y. Yasui, Supersymmetric nonlinear $\sigma$-model $O N E_{8} / \mathrm{SO}(10) \times \mathrm{SU}(3) \times \mathrm{U}(1), Z$. Phys. C 29 (1985) 123 [INSPIRE].

[6] M. Ibe, T. Moroi and T.T. Yanagida, Possible Signals of Wino LSP at the Large Hadron Collider, Phys. Lett. B 644 (2007) 355 [hep-ph/0610277] [INSPIRE].

[7] M. Ibe and T.T. Yanagida, The Lightest Higgs Boson Mass in Pure Gravity Mediation Model, Phys. Lett. B 709 (2012) 374 [arXiv:1112.2462] [INSPIRE].

[8] N. Arkani-Hamed, A. Gupta, D.E. Kaplan, N. Weiner and T. Zorawski, Simply Unnatural Supersymmetry, arXiv:1212.6971 [INSPIRE].

[9] G.F. Giudice, M.A. Luty, H. Murayama and R. Rattazzi, Gaugino mass without singlets, JHEP 12 (1998) 027 [hep-ph/9810442] [INSPIRE].

[10] L. Randall and R. Sundrum, Out of this world supersymmetry breaking, Nucl. Phys. B 557 (1999) 79 [hep-th/9810155] [INSPIRE].

[11] K. Inoue, M. Kawasaki, M. Yamaguchi and T. Yanagida, Vanishing squark and slepton masses in a class of supergravity models, Phys. Rev. D 45 (1992) 328 [INSPIRE].

[12] K. Harigaya, T.T. Yanagida and N. Yokozaki, Seminatural SUSY from the $E_{7}$ nonlinear $\sigma$-model, PTEP 2015 (2015) $083 \mathrm{~B} 03$ [arXiv:1504.02266] [INSPIRE].

[13] W. Yin and N. Yokozaki, Splitting Mass Spectra and Muon g-2 in Higgs-Anomaly Mediation, arXiv:1607.05705 [INSPIRE].

[14] M. Fukugita and T. Yanagida, Baryogenesis Without Grand Unification, Phys. Lett. B 174 (1986) 45 [INSPIRE].

[15] W. Buchmüller, R.D. Peccei and T. Yanagida, Leptogenesis as the origin of matter, Ann. Rev. Nucl. Part. Sci. 55 (2005) 311 [hep-ph/0502169] [inSPIRE].

[16] S. Davidson, E. Nardi and Y. Nir, Leptogenesis, Phys. Rept. 466 (2008) 105 [arXiv:0802.2962] [INSPIRE].

[17] Z. Komargodski and N. Seiberg, Comments on Supercurrent Multiplets, Supersymmetric Field Theories and Supergravity, JHEP 07 (2010) 017 [arXiv: 1002.2228] [INSPIRE].

[18] T. Kugo and T.T. Yanagida, Coupling Supersymmetric Nonlinear $\sigma$-models to Supergravity, Prog. Theor. Phys. 124 (2010) 555 [arXiv:1003.5985] [InSPIRE].

[19] T. Goto and T. Yanagida, Nonlinear $\sigma$-model coupled to a broken supergravity, Prog. Theor. Phys. 83 (1990) 1076 [INSPIRE].

[20] M. Yamaguchi and W. Yin, A Novel Approach to Fine-Tuned Supersymmetric Standard Models - Case of Non-Universal Higgs Masses model, arXiv: 1606.04953 [INSPIRE].

[21] Y. Okada, M. Yamaguchi and T. Yanagida, Upper bound of the lightest Higgs boson mass in the minimal supersymmetric standard model, Prog. Theor. Phys. 85 (1991) 1 [INSPIRE].

[22] J.R. Ellis, G. Ridolfi and F. Zwirner, Radiative corrections to the masses of supersymmetric Higgs bosons, Phys. Lett. B 257 (1991) 83 [INSPIRE].

[23] H.E. Haber and R. Hempfling, Can the mass of the lightest Higgs boson of the minimal supersymmetric model be larger than $m(Z)$ ?, Phys. Rev. Lett. 66 (1991) 1815 [INSPIRE]. 
[24] Y. Okada, M. Yamaguchi and T. Yanagida, Renormalization group analysis on the Higgs mass in the softly broken supersymmetric standard model, Phys. Lett. B 262 (1991) 54 [INSPIRE].

[25] J.R. Ellis, G. Ridolfi and F. Zwirner, On radiative corrections to supersymmetric Higgs boson masses and their implications for LEP searches, Phys. Lett. B 262 (1991) 477 [InSPIRE].

[26] D.M. Pierce, J.A. Bagger, K.T. Matchev and R.-j. Zhang, Precision corrections in the minimal supersymmetric standard model, Nucl. Phys. B 491 (1997) 3 [hep-ph/9606211] [INSPIRE].

[27] K. Harigaya, T.T. Yanagida and N. Yokozaki, Higgs boson mass of $125 \mathrm{GeV}$ and $g-2$ of the muon in a gaugino mediation model, Phys. Rev. D 91 (2015) 075010 [arXiv:1501.07447] [INSPIRE].

[28] L.J. Hall, R. Rattazzi and U. Sarid, The Top quark mass in supersymmetric $\mathrm{SO}(10)$ unification, Phys. Rev. D 50 (1994) 7048 [hep-ph/9306309] [INSPIRE].

[29] M. Carena, M. Olechowski, S. Pokorski and C.E.M. Wagner, Electroweak symmetry breaking and bottom-top Yukawa unification, Nucl. Phys. B 426 (1994) 269 [hep-ph/9402253] [INSPIRE].

[30] A. Djouadi, J.-L. Kneur and G. Moultaka, SuSpect: A Fortran code for the supersymmetric and Higgs particle spectrum in the MSSM, Comput. Phys. Commun. 176 (2007) 426 [hep-ph/0211331] [INSPIRE].

[31] CMS collaboration, Searches for long-lived charged particles in pp collisions at $\sqrt{s}=7$ and $8 \mathrm{TeV}$, JHEP 07 (2013) 122 [arXiv: 1305. 0491] [INSPIRE].

[32] ATLAS collaboration, Further searches for squarks and gluinos in final states with jets and missing transverse momentum at $\sqrt{s}=13 \mathrm{TeV}$ with the ATLAS detector,

ATLAS-CONF-2016-078 (2016).

[33] ATLAS collaboration, Search for charginos nearly mass degenerate with the lightest neutralino based on a disappearing-track signature in pp collisions at $\sqrt{s}=8 \mathrm{TeV}$ with the ATLAS detector, Phys. Rev. D 88 (2013) 112006 [arXiv:1310.3675] [INSPIRE].

[34] B. Bhattacherjee, M. Ibe, K. Ichikawa, S. Matsumoto and K. Nishiyama, Wino Dark Matter and Future dSph Observations, JHEP 07 (2014) 080 [arXiv:1405.4914] [INSPIRE].

[35] A.D. Box and X. Tata, Threshold and Flavour Effects in the Renormalization Group Equations of the MSSM II: Dimensionful couplings, Phys. Rev. D 79 (2009) 035004 [Erratum ibid. D 82 (2010) 119905] [arXiv:0810.5765] [INSPIRE].

[36] J. Pardo Vega and G. Villadoro, SusyHD: Higgs mass Determination in Supersymmetry, JHEP 07 (2015) 159 [arXiv: 1504.05200] [INSPIRE].

[37] ATLAS collaboration, Search for Supersymmetry at the high luminosity LHC with the ATLAS experiment, ATL-PHYS-PUB-2014-010 (2014).

[38] G.F. Giudice, A. Notari, M. Raidal, A. Riotto and A. Strumia, Towards a complete theory of thermal leptogenesis in the SM and MSSM, Nucl. Phys. B 685 (2004) 89 [hep-ph/0310123] [INSPIRE].

[39] W. Buchmüller, P. Di Bari and M. Plümacher, Leptogenesis for pedestrians, Annals Phys. 315 (2005) 305 [hep-ph/0401240] [INSPIRE]. 
[40] Muon G-2 collaboration, G.W. Bennett et al., Final Report of the Muon E821 Anomalous Magnetic Moment Measurement at BNL, Phys. Rev. D 73 (2006) 072003 [hep-ex/0602035] [INSPIRE].

[41] B.L. Roberts, Status of the Fermilab Muon $(g-2)$ Experiment, Chin. Phys. C 34 (2010) 741 [arXiv: 1001.2898] [INSPIRE].

[42] K. Hagiwara, R. Liao, A.D. Martin, D. Nomura and T. Teubner, $(g-2)_{\mu}$ and $\alpha\left(M_{Z}^{2}\right)$ re-evaluated using new precise data, J. Phys. G 38 (2011) 085003 [arXiv:1105.3149] [INSPIRE].

[43] M. Davier, A. Hoecker, B. Malaescu and Z. Zhang, Reevaluation of the Hadronic Contributions to the Muon g-2 and to alpha(MZ), Eur. Phys. J. C 71 (2011) 1515 [Erratum ibid. C 72 (2012) 1874] [arXiv: 1010.4180] [INSPIRE].

[44] J.L. Lopez, D.V. Nanopoulos and X. Wang, Large $(g-2)_{\mu}$ in $\mathrm{SU}(5) \times \mathrm{U}(1)$ supergravity models, Phys. Rev. D 49 (1994) 366 [hep-ph/9308336] [INSPIRE].

[45] U. Chattopadhyay and P. Nath, Probing supergravity grand unification in the Brookhaven g-2 experiment, Phys. Rev. D 53 (1996) 1648 [hep-ph/9507386] [InSPIRE].

[46] T. Moroi, The Muon anomalous magnetic dipole moment in the minimal supersymmetric standard model, Phys. Rev. D 53 (1996) 6565 [Erratum ibid. D 56 (1997) 4424] [hep-ph/9512396] [INSPIRE].

[47] S. Marchetti, S. Mertens, U. Nierste and D. Stöckinger, $\tan \beta$-enhanced supersymmetric corrections to the anomalous magnetic moment of the muon, Phys. Rev. D 79 (2009) 013010 [arXiv:0808.1530] [INSPIRE].

[48] G. Degrassi and G.F. Giudice, QED logarithms in the electroweak corrections to the muon anomalous magnetic moment, Phys. Rev. D 58 (1998) 053007 [hep-ph/9803384] [INSPIRE].

[49] M. Endo, K. Hamaguchi, S. Iwamoto and N. Yokozaki, Higgs Mass and Muon Anomalous Magnetic Moment in Supersymmetric Models with Vector-Like Matters, Phys. Rev. D 84 (2011) 075017 [arXiv: 1108.3071] [INSPIRE].

[50] T. Moroi, R. Sato and T.T. Yanagida, Extra Matters Decree the Relatively Heavy Higgs of Mass about $125 \mathrm{GeV}$ in the Supersymmetric Model, Phys. Lett. B 709 (2012) 218 [arXiv:1112.3142] [INSPIRE].

[51] M. Endo, K. Hamaguchi, S. Iwamoto and N. Yokozaki, Higgs mass, muon g-2 and LHC prospects in gauge mediation models with vector-like matters, Phys. Rev. D 85 (2012) 095012 [arXiv: 1112.5653] [INSPIRE].

[52] M. Endo, K. Hamaguchi, S. Iwamoto, K. Nakayama and N. Yokozaki, Higgs mass and muon anomalous magnetic moment in the U(1) extended MSSM, Phys. Rev. D 85 (2012) 095006 [arXiv:1112.6412] [INSPIRE].

[53] K. Nakayama and N. Yokozaki, Peccei-Quinn extended gauge-mediation model with vector-like matter, JHEP 11 (2012) 158 [arXiv:1204.5420] [INSPIRE].

[54] R. Sato, K. Tobioka and N. Yokozaki, Enhanced Diphoton Signal of the Higgs Boson and the Muon g-2 in Gauge Mediation Models, Phys. Lett. B 716 (2012) 441 [arXiv:1208.2630] [INSPIRE].

[55] Y. Shimizu and W. Yin, Natural split mechanism for sfermions: $N=2$ supersymmetry in phenomenology, Phys. Lett. B 754 (2016) 118 [arXiv:1509.04933] [INSPIRE]. 
[56] S. Heinemeyer, W. Hollik and G. Weiglein, FeynHiggs: A Program for the calculation of the masses of the neutral CP even Higgs bosons in the MSSM, Comput. Phys. Commun. 124 (2000) 76 [hep-ph/9812320] [INSPIRE].

[57] S. Heinemeyer, W. Hollik and G. Weiglein, The Masses of the neutral CP-even Higgs bosons in the MSSM: Accurate analysis at the two loop level, Eur. Phys. J. C 9 (1999) 343 [hep-ph/9812472] [INSPIRE].

[58] G. Degrassi, S. Heinemeyer, W. Hollik, P. Slavich and G. Weiglein, Towards high precision predictions for the MSSM Higgs sector, Eur. Phys. J. C 28 (2003) 133 [hep-ph/0212020] [INSPIRE].

[59] M. Frank, T. Hahn, S. Heinemeyer, W. Hollik, H. Rzehak and G. Weiglein, The Higgs Boson Masses and Mixings of the Complex MSSM in the Feynman-Diagrammatic Approach, JHEP 02 (2007) 047 [hep-ph/0611326] [INSPIRE].

[60] T. Hahn, S. Heinemeyer, W. Hollik, H. Rzehak and G. Weiglein, High-Precision Predictions for the Light CP -Even Higgs Boson Mass of the Minimal Supersymmetric Standard Model, Phys. Rev. Lett. 112 (2014) 141801 [arXiv:1312.4937] [INSPIRE].

[61] M. Endo, K. Hamaguchi, T. Kitahara and T. Yoshinaga, Probing Bino contribution to muon $g-2$, JHEP 11 (2013) 013 [arXiv: 1309.3065] [INSPIRE].

[62] ACME collaboration, J. Baron et al., Order of Magnitude Smaller Limit on the Electric Dipole Moment of the Electron, Science 343 (2014) 269 [arXiv:1310.7534] [InSPIRE]. 